\title{
The histochemistry of thiols and disulphides. II. Methodology of differential staining
}

\author{
T. O. SIPPEL \\ Department of Anatomy, University of Michigan, Ann Arbor, Michigan 48109, USA
}

Received 23 February 1978

Synopsis. The reduction of disulphide bonds by various mercaptans and tri- $n$-butylphosphine (TBP) has been examined in paraffin sections of rat tissues. A 're-reduction' procedure demonstrating any residual disulphides shows that nearly equivalent endpoints are reached by all of the reagents at $\mathrm{pH} 8.5$ and room temperature, though at greatly differing rates. TBP is the reductant of choice in that it acts rapidly, cannot cause the thiolation which is more or less pronounced with certain mercaptans and least reverses the prior alkylation of native thiol groups by iodoacetate or $\mathrm{N}$-substituted malemides. Supporting studies establish that, except in highly compact structures, native as well as generated thiol groups can be visualized with satisfactory completeness and specificity by $\mathrm{N}$-(4-aminophenyl)maleimide followed by a diazotization and coupling sequence. These findings provide the basis for the selective staining of disulphides, either alone or differentiated from native thiols in the same section.

\section{Introduction}

Danielli (1950) suggested that protein disulphide bonds in tissue sections might be visualized, after the blocking of pre-existing thiol* and other reactive groups with phenyl isocyanate, by means of their cyanolysis to thiols; these he proposed to label with dinitrofluorobenzene. Although this particular procedure turned out to be impractical (Pearse, 1968), the principle was applied successfully by Barrnett \& Seligman (1954) who used iodoacetate or N-ethylmaleimide (NEM) to prevent subsequent reaction of the native thiols with their more specific 'DDD' thiol staining reagent. They recognized that, at most, half of the available thiol groups were liberated from disulphide bonds split by cyanide but noted that more powerful agents reversed the alkylating blockades. Their alternative was to stain native and generated thiols together after reducing the disulphides with a hot alkalinized mercaptan solution;

\footnotetext{
*The term thiol refers to a tissue constituent bearing the sulphydryl or, loosely, thiol group and mercaptan designates a sulphydryl compound applied as a reagent.
}

(C) 1978 Chapman and Hall Ltd. Printed in Great Britain. 
despite requiring comparison with thiol staining alone to localize or measure disulphides 'by difference', the procedure remains in use (for example, Cowden \& Curtis, 1975).

This inquiry into the possibility of demonstrating disulphide bonds selectively as well as quantitatively stems from the observation (Sippel, 1973) that the bound form of the chromogenic thiol reagent $\mathrm{N}$-(4-aminophenyl)maleimide (APM), when labelled on the section by diazotization and coupling, resisted overnight exposures to several different mercaptans at $\mathrm{pH} 8$ and room temperature. Proposed as a point of departure for differentiating disulphides from thiols in the same section (dual staining), the finding also implied that disulphide sites alone could be shown after the native thiois were blocked by a monofunctional maleimide (single staining).

The initial studies undertaken to implement both approaches concern completeness of the blocking of thiol groups by various maleimides (and iodoacetate) and, with regard to their visualization, the specificity of APM; in this connection, the conditions for stoichiometric labelling of the APM-thiol adducts are examined with special attention to the blue coupler 'Nitro Red' (Sippel, 1973), now purified to correct its earlier fault of yielding two colours. Next, the rates and extents of disulphide reduction by not only different mercaptans but also tri- $n$-butylphosphine (TBP) are compared; the endpoint tests used for this purpose prove sensitive enough to reveal slight departures from specificity arising largely from the reversal of thiol blockade even under mild conditions of reduction.

Standard methods devised in the light of these studies are offered in the next paper along with illustrations of typical results obtained on paraffin sections of rat tissues; reference to both may be helpful. Critical examination of the special fixation procedure introduced below will be deferred.

\section{Materials and methods}

\section{CHEMICALS}

\section{Thiol reagents}

The synthesis of APM (Sippel, 1973) was improved mainly by starting with $p$-phenylenediamine recrystallized from ethanol until pale pink. After drying in vacuo its reaction with one equivalent of maleic anhydride, in $10 \%$ larger volumes than used earlier, consistently gave the desired bright orange unhydrated 4-aminomaleanilic acid in very high yield. This was cyclodehydrated as before but the extract in EtOAc was dried with $\mathrm{MgSO}_{4}$ and evaporated under reduced pressure. APM obtained by filtering, concentrating and cooling the hot toluene extract of the residue was recrystallized from toluene as bright orange crystals, m.p. $172-176^{\circ} \mathrm{C}$ (uncorrected) in an average yield $23 \%$ of theoretical. Stored dry at room temperature, it has been stable for over three years.

The fluorogenic reagent $\mathrm{N}-(1-$ anilinonaphthyl-4)maleimide (ANM) was prepared as suggested by Kanaoka et al. (1973), though in only $11 \%$ yield. $\mathrm{N}$-phenyl- and $\mathrm{N}$-benzylmaleimide were synthesized by standard methods, whereas NEM was purchased from Nutritional Biochemicals (Cleveland, Ohio, USA). Iodoacetic acid from the same source was recrystallized from cyclohexane and stored in the cold. Formamidine disulphide dihydrochloride prepared according to Toennies (1973) was kept cold and dry. 


\section{Azo couplers}

1-Amino-8-hydroxy-2-(4'nitrobenzeneazo)naphthalene-3,6-disulphonic acid was prepared in $0.1 \mathrm{M}$ quantity from the directions of Fierz-David \& Blangey (1949, p. 288). After the crude product was washed with $2 \mathrm{~N} \mathrm{HCl}$ and dissolved in $500 \mathrm{ml}$ water with $8 \mathrm{~g} \mathrm{NaOH}$, the solution was neutralized with HOAc and stirred with $150 \mathrm{~g} \mathrm{NaOAc}$ as it was heated to $50^{\circ} \mathrm{C}$. The cooled precipitate was collected and washed with half-saturated $\mathrm{NaOAc}$ followed by $95 \%$ ethanol. Soxhlet extraction of the dried pulverized cake with $95 \%$ ethanol left $45 \mathrm{~g}$ of the disodium salt 'Nitro Red' as a bluish-black powder. Thin layer chromatography (phenol: ethanol: water 4:3:4 on Merck silica gel $\mathrm{GF}_{25}$ sheets) showed a dominant bluish-red component of $\mathrm{R}_{\mathrm{F}} 0.54$; the spot turned dull blue in ammonia fumes and when sprayed with aqueous Fast Red GG became a pH-insensitive blue colour best seen after removal of the orange background with ether. Unreactive contaminants were a blue disazo compound $\left(\mathrm{R}_{\mathrm{F}}\right.$ $0.60)$ and an unknown red spot $\left(R_{F} 0.56\right)$. Residual $H$-acid $\left(R_{F} 0.47\right.$ and coupling purple to Fast Red GG) could not be detected; it apparently was responsible for the extraneous red coupling to diazotized bound APM (Sippel, 1973).

Other coupling compounds used were $\mathrm{N}$-naphthylethylenediamine dihydrochloride (K\& K Laboratories, Plainview, New York, USA) and, obtained from Aldrich Chemical Co. (Milwaukee, Wisconsin, USA), chromotropic acid as well as technical grade $\mathrm{N}$-phenyl-1-naphthylamine which was recrystallized from ethanol.

\section{Reducing agents}

Benzyl mercaptan, $n$-dodecyl mercaptan and 2,3-dimercaptopropanol ('BAL' = British anti-Lewisite) were obtained from K \& K Laboratories, 1-thioglycerol and 1,4-dithiobutane from Aldrich Chemical Co., and 2-mercaptoethanol, dithiothreitol and thioglycolic acid from Nutritional Biochemicals; some of the last was re-distilled $\left(104^{\circ} \mathrm{C}\right.$ at $12 \mathrm{~mm} \mathrm{Hg}$ ) and stored at $5^{\circ} \mathrm{C}$. TBP was obtained from Aldrich and from BDH Chemicals Ltd. via Gallard-Schlesinger Corp. (Carle Place, New York, USA) and likewise kept cold.

\section{PROCEDURES}

General

As described fully in the next paper, composite $6 \mu \mathrm{m}$ paraffin sections of seven rat organs (eye, brain, tongue, liver, kidney, duodenum and pancreas) that had been fixed in neutral formalin containing an organomercurial were deparaffinized and exposed to dilute acidified mercaptoethanol to dissociate the protective mercaptides. Subsequent treatment varied according to the nature of the test but, unless specified otherwise, thiol groups ultimately were stained as before (Sippel, 1973) except that the diazotized bound APM was labelled blue in one step by coupling it to $1 \%$ Nitro Red in $0.1 \mathrm{M}$ sodium phosphate buffer at $\mathrm{pH} 7$ for 2 min; after a water rinse, direct staining by the Nitro Red was removed by differentiating the sections for $5 \min$ in $1 \%(\mathrm{v} / \mathrm{v})$ triethylamine in $70 \%$ ethanol. The dehydrated and cleared sections were mounted in DPX (BDH Chemicals, Ltd.).

\section{Colour development}

For study of the variables in the labelling process, native thiol groups first were reacted with APM. Diazotization time was determined by exposing the sections to $0.05 \mathrm{~N}$ 
$\mathrm{HNO}_{2}$ for different periods before coupling to Nitro Red, after which any remaining undiazotized APM was shown by the red coloration added when the sections were rediazotized for $5 \mathrm{~min}$ and coupled to $0.5 \%$ chromotropic acid in $0.1 \mathrm{M}$ sodium acetate- $\mathrm{HCl}$ buffer at $\mathrm{pH} 5$ for 2 min. Diazonium stability was judged by holding fully diazotized sections under different conditions (see Results) before azo coupling was carried out by their transfer directly into $0.25 \% \mathrm{~N}$-naphthylethylenediamine in $0.1 \mathrm{~N}$ $\mathrm{HCl}$ for $2 \mathrm{~min}$; to convert the resulting orange label into its magenta hydrochloride, the sections were dehydrated in two changes of acetone containing one drop of concentrated $\mathrm{HCl}$ in $40 \mathrm{ml}$. Coupling times under these same conditions were found by labelling any uncoupled diazonium groups in a distinctive colour: after various periods of coupling to Nitro Red the sections were placed in N-naphythylenediamine as above; after coupling to chromotropic acid the sections were transferred to $1 \% \mathrm{~N}$-phenyl-1. naphthylamine in acetic acid for $2 \mathrm{~min}$ and the resulting label was converted to its violet hydrochloride.

\section{Thiol blockade}

The gradual extinction of standard thiol staining was used to indicate the course of nonchromogenic alkylation of native thiol groups. Various concentrations of the three monofunctional maleimides were applied at $\mathrm{pH} 6$ in the solvent mixture used for APM (Sippel, 1973), whereas iodoacetic acid was dissolved at twice the desired final strength in one volume of $0.05 \mathrm{M}$ sodium barbital, adjusted to $\mathrm{pH} 8.5$ with strong $\mathrm{NaOH}$ and then diluted with one volume of 2-propanol.

In similar fashion, the action of $5 \mathrm{mM}$ APM was followed by staining the residual thiols for $1 \mathrm{~h}$ with $0.5 \mathrm{mM}$ ANM dissolved in the $\mathrm{pH} 6$ solvent. The sections were examined for bright blue fluorescence under darkfield u.v. illumination (GE H85 A3/UV lamp and Corning 7-47 filter). Afterwards the coverslips were removed and the bound APM was labelled blue in the usual way. This procedure was carried out both on a series of sections containing native thiols and on another with thiols generated by the reduction of disulphide bonds after the native groups were blocked by NEM.

To demonstrate non-specific staining when APM was applied for increasing times and then labelled blue, the same two types of sections as used above were pretreated for $5 \mathrm{~min}$ with fresh $1 \%$ formamidine disulphide in $0.1 \mathrm{~N} \mathrm{HCl}$ to block only their thiol groups. The solution was discarded promptly to avoid an intractable precipitate on the glassware.

\section{Disulphide reduction}

Mercaptans applied as reductants at arbitrary concentrations (see Results) were, except for thioglycolic acid which was handled in the same way as iodoacetic acid, dissolved in 2-propanol followed by an equal volume of $0.05 \mathrm{M}$ sodium barbital- $\mathrm{HCl}$ buffer of $\mathrm{pH}$ 8.5. TBP was used in nearly saturated $(0.25 \% \mathrm{v} / \mathrm{v})$ solutions in this solvent mixture as well as in 50\% 2-propanol buffered to $\mathrm{pH} 6$ and 7 with $0.05 \mathrm{M}$ phosphate.

The 're-reduction' procedure employed to study the parameters of reduction essentially revealed any unreduced disulphides. In general, after sections had been reduced and the resulting thiols blocked along with the native groups, they were reduced again and stained for any newly generated thiol groups. When the test was 
utilized to determine the time course of reduction, a given agent was applied as the first reductant to a series of sections for different times followed by overnight alkylation with NEM; to visualize remaining disulphides the sections were re-reduced in $0.1 \%$ dithiothreitol for $1 \mathrm{~h}$ before staining. For comparing the relative extents of reduction by any two agents, they were applied as the first and second reductants, respectively, for about half again as long as each required to reach its endpoint; again the intervening blockade was imposed by NEM. To study reversal of blockade during second reductions proceeding for the same relative times as above, the first reductant was either dithiothreitol (before re-reduction by any mercaptan) or TBP (before TBP) applied for at least $1 \mathrm{~h}$. The stability of NEM blockade was tested toward all reductants whereas that imposed by other alkylators was noted only during re-reductions by benzyl mercaptan, dithiothreitol and TBP. When the blocking agent was APM it was applied for just $1 \mathrm{~h}$ and labelled either red (chromotropic acid) or blue (Nitro Red), hence the final thiol staining after re-reduction was developed to the contrasting colour.

\section{Results}

\section{Colour development}

APM bound to thiol groups was completely diazotized in less than 1 min except in the lens nucleus and the prekeratin of lingual papillae. Diazotization in these two sites of exceptionally compact protein seemed still incomplete at 5 min but the apparent lag later was traced to carryover of diazonium groups which had failed to couple to Nitro Red. Cold was unnecessary inasmuch as the diazonium groups were stable for at least $10 \mathrm{~min}$ in 0.01 and $0.1 \mathrm{~N} \mathrm{HCl}$ at room temperature. The weaker was selected for rinsing to minimize changes in the buffered coupling solutions that ordinarily followed. Treatment with sulphamic acid to prevent carryover of $\mathrm{HNO}_{2}$ was not required with the present couplers.

Coupling of the diazotized bound APM to $0.5 \%$ chromotropic acid at pH 5 was not quite complete in compact tissues in $5 \mathrm{~min}$ but finished elsewhere within $15 \mathrm{~s}$. Because in such a short time there was no detectable loss of diazonium reactivity in the absence of a coupler, formation of the red label in typical tissues was presumed to be quantitative.

At pH 5 the coupling to Nitro Red, although rapid in erythrocytes and keratohyaline granules, was relatively slow in most sites and required nearly $10 \mathrm{~min}$ to reach its full level in not only compact tissues but also myelinated structures and, inexplicably, the cytoplasm of proximal kidney tubules. By this time diazotized sections held in pH 5 buffer lacking a coupler were estimated to have lost nearly half of their coupling capacity, although little further decrease occurred in the course of $1 \mathrm{~h}$. At $\mathrm{pH} 6$ the balance was shifted markedly toward coupling, and at $\mathrm{pH} 7$ blue labelling in noncompact tissues was complete within $15 \mathrm{~s}$ in which time only about a tenth of the diazonium groups initially present lost reactivity in the absence of a coupler; cooling in ice slowed this inactivation but left coupling still incomplete at $30 \mathrm{~min}$.

The implied departure from stoichiometric blue labelling of the thiols in any site poorly penetrated by Nitro Red was noted to be a potential source of non-specific 
labelling of disulphides in dual staining: most of the diazonium groups that lost their coupling capacity when sections were held at $\mathrm{pH} 7$ could be reduced back to the arylamine level, that is, to bound APM, unless further decomposed at a high $\mathrm{pH}$. Thus in dual staining the reduction of disulphides should follow the alkaline bath needed to complete the extraction of the polychrome direct staining of sections by Nitro Red. In this regard, components stained blue by the dye eventually decolorized in water alone, whereas only treatments with such as triethylamine in ethanol removed red staining from connective tissue fibres; it proved more effective than either aqueous $\mathrm{Li}_{2} \mathrm{CO}_{3}$ or ammonia for this purpose. An alkali-fast violet acidophila restricted to unidentified free cells most common in the duodenal connective tissue was almost entirely extractable by further differentiation of the sections in acid alcohol.

\section{Colourless blockade of native thiols}

Apart from faint staining in the lens nucleus, the reactivity of sections toward APM $(5 \mathrm{mM}, 1 \mathrm{~h}$ ) was abolished by their prior exposure to fresh $10 \mathrm{~mm}$ solutions of any of the three monofunctional maleimides for at least $4 \mathrm{~h}$. Overnight treatments allowed the use of solutions kept at room temperature for up to one week. The time required for total blocking could be shortened to $1 \mathrm{~h}$ with fresh $100 \mathrm{mM} \mathrm{NEM}, 50 \mathrm{mM}$ $\mathrm{N}$-phenylmaleimide or $20 \mathrm{mM} \mathrm{N}$-benzylmaleimide; since the last of these was difficult to synthesize and expensive if bought, it was not studied further.

Pre-treatment with $100 \mathrm{~mm}$ iodoacetate for $1-4 \mathrm{~h}$ left a low level of thiol stainability of about the same intensity as after blocking by formamidine disulphide (see below). When applied overnight, 10 or $20 \mathrm{mM}$ iodoacetate was quite effective but only 50 and $100 \mathrm{~mm}$ solutions blocked as thoroughly as the maleimides.

\section{Chromogenic thiol blockade}

The thiols generated by reduction of disulphide bonds (after overnight alkylation of the sections by NEM) reacted completely with $5 \mathrm{mM}$ APM within $30 \mathrm{~min}$, as shown by the absence of fluorescence above autofluorescence upon subsequent staining by ANM. Accordingly, when the bound APM was visualized by diazotization and coupling, there was no difference in colour between sections that had been exposed to APM for 30 and $60 \mathrm{~min}$. The specificity of staining was satisfactory with such short treatments inasmuch as, in other sections in which the generated thiols were specifically blocked by formamidine disulphide, no colour could be developed after a $30 \mathrm{~min}$ exposure to APM and barely any was visible after $1 \mathrm{~h}$. After 2 and $4 \mathrm{~h}$, however, increasing staining was noted in disulphide-rich structures, especially lingual keratin, Descemet's membrane and the lens capsule. Being stronger than their levels of non-specific staining (see below), it indicated slow reversal of the formamidine disulphide blockade; some colour developable in erythrocytes after long exposures to APM probably represented its reaction with non-thiol groups that failed to be blocked by the initial NEM treatment.

The use of APM to visualize native thiol groups was less straightforward. Whereas in most parts of the sections those groups demonstrable by fluorescent overlay with ANM were blocked fully by APM applied for $60 \mathrm{~min}$ and very nearly so in $30 \mathrm{~min}$, even a $4 \mathrm{~h}$ exposure to APM failed to prevent bright blue fluorescence in compact structures. By this time, however, the colour developed by diazotization and coupling 
of the bound APM was significant in Descemet's membrane and the lens capsule. It was identical to the non-specific staining of these structures in other sections that had been blocked by formamidine disulphide. In either circumstance, they were uncoloured when the exposure to APM was limited to $1 \mathrm{~h}$ at which time, in blocked sections, staining elsewhere was only just detectable except in ery throcytes.

In a dual staining procedure, the presumed consequence of the incomplete reaction of APM with the thiols of compact structures would be their later mislabelling as disulphides. That an unexpected course of carryover could ensue because of the intervening diazotization step was discovered after the inadvertent exposure of some ordinary sections to $\mathrm{HNO}_{2}$ before APM. All thiol staining was abolished but it became evident that the thiols had become nitrosated rather than oxidized (cf. Saville, 1958) when their reactivity toward APM was shown to be regenerated by chloromercuration $\left(0.1 \% \mathrm{HgCl}_{2}\right.$ in $0.1 \mathrm{~N} \mathrm{HCl}$ for $\left.1 \mathrm{~min}\right)$ followed by routine dissociation of mercaptides in acidified mercaptoethanol. Inasmuch as the S-nitroso groups also were reducible by both mercaptans and TBP to free thiols, these still would be indistinguishable from the thiols generated from disulphides.

\section{Reduction by mercaptans}

Upon a second reduction by dithiothreitol for $1 \mathrm{~h}$, the same minimal level of thiol stainability was reached in all sections that had been reduced before NEM blockade for at least $15 \mathrm{~min}$ in $0.1 \%$ dithiothereitol or 1,4-dithiobutane; $1 \mathrm{~h}$ in $1 \%$ benzyl mercaptan, BAL or redistilled thioglycolic acid; $2 \mathrm{~h}$ in saturated (approximately $0.1 \%$ ) $n$-dodecyl mercaptan or $1 \%$ mercaptoethanol; and about $4 \mathrm{~h}$ in $2 \%$ thioglycerol (all but dithiothreitol are liquids and their concentrations are given in volume percentages). These then were the shortest effective treatments by the respective mercaptan solutions and resulted in reduction to comparable extents.

Moderate staining was produced in the above trials no matter how long a first reduction by dithiothreitol or any other mercaptan had been allowed to run. There was less colour when the second reduction by dithiothreitol was shortened to $25 \mathrm{~min}$. However, its cause did not begin to emerge until the remaining mercaptans, likewise applied for about half again as long as they required to reduce disulphides fully, were tested as second reducants.

Re-reduction by benzyl mercaptan, mercaptoethanol, thioglycerol, $n$-dodecyl mercaptan, or purified thioglycolate produced pale stainability confined to disulphiderich structures. However, the implied re-oxidation of thiol pairs before they could be alkylated by the NEM contributed little to the final staining: two cycles of first reduction and alkylation decreased the colour only slightly; and holding the reduced sections in the maleimide solvent for $1 \mathrm{~h}$ before blocking caused no detectable increase. Since these same structures were also the darkest in other sections simply stained for the sum of thiols and disulphides (which together comprise the blocked groups in re-reduction tests) some instability of the NEM blockade seemed indicated. It was supported by the finding that the amount of staining varied with the nature of the blocking agent, with re-reduction by benzyl mercaptan yielding more final colour in sections blocked by phenylmaleimide and less in those blocked by iodoacetate.

Dithiothreitol, dithiobutane, and BAL all induced more stainability in NEMblocked sections than did any monomercaptan and, in the case of dithiothreitol, the 
same effect occurred in sections blocked by phenylmaleimide or iodoacetate. Amongst disulphide-rich structures the internal elastic laminae of arteries in particular showed extra staining but some occurred in cytoplasm as well. It probably arose from thiolation which, proceeding by a different mechanism (see Discussion), certainly would account for the effect of unpurified thioglycolic acid: re-reduction by this reagent induced very strong staining not only throughout the sections but even in the gelatin coatings on the slides.

Some reversibility of thiol alkylation by APM also was evident in that re-reduction by benzyl mercaptan of sections containing either blue- or red-labelled bound APM caused slight overall shading by the converse colour developed in the final staining procedure. Again, dithiothreitol had a more pronounced effect. In both cases compact structures strongly assumed the second colour, although not so much in the lens nucleus as in lingual papillae. No fading of the first colour could be detected in control sections submitted to re-reduction procedures with APM omitted from the final staining solution.

\section{Reduction by $T B P$}

Whether at $\mathrm{pH} 6,7$ or 8.5 , TBP required about 20 min to reach an endpoint in the reduction of disulphide bonds. However, the extent of reduction increased with $\mathrm{pH}$. So also did the degree of reversal of NEM blockade when the reagent was applied as a second reductant for $30 \mathrm{~min}$; nonetheless it was smaller at the routinely adopted $\mathrm{pH}$ of 8.5 than that caused by any monomercaptan. As had been found with benzyl mercaptan, TBP reversed blocking by iodoacetate least and by phenylmaleimide most, although the differences were barely detectable. It also caused about the same shifts in colour of sections containing labelled APM but, since some changes occurred even when APM was omitted from the final staining procedure, part of the reversal could be attributed to reduction of the azo bonds of the labels, that is, to regeneration of bound APM.

Until TBP was applied as a first reductant for $1 \mathrm{~h}$ it was not as effective as either dithiothreitol or benzyl mercaptan in reducing the disulphide bonds in the myelinated tracts of the brain and the outer segments of retinal rods. Conversely, neither of the mercaptans reduced the bonds in pancreatic zymogen granules as thoroughly as did TBP in $30 \mathrm{~min}$; in the case of dithiothreitol a first reduction for as long as $1 \mathrm{~h}$ still left some of these disulphides demonstrable upon re-reduction by TBP.

Working solutions of TBP seemed to retain their effectiveness for several days unless contaminated by Nitro Red extracted from sections. However, it was thought better to prepare a fresh solution on the day of use.

\section{Discussion}

A primary requirement for visualizing disulphides as thiols is reproducible stoichiometry in their reduction. Strongly alkaline cyanolysis yields less than its theoretical half-disulphide equivalent (Pearse, 1968), whereas sulphitolysis, though only infrequently tried (for example, Foraker \& Wingo, 1956), seems complete when carried out with organomercurial trapping of the thiol group at a neutral $\mathrm{pH}$ (Sippel, unpublished work). Nevertheless, total reduction of each bond to two thiol groups is preferable for maximum visibility. 
Reduction by sulphydryl-disulphide interchange in an excess of a mercaptan at up to $50^{\circ} \mathrm{C}$ has been favoured by histochemists since the pioneering work of Barrnett $\&$ Seligman (1954). They had judged aqueous thioglycolate to be more effective than, for example, mercaptoethanol (but inferior to BAL) applied in acetone with pyridine and recommended its use. Uncertainties attending the differing conditions prompted the present survey of these three mercaptans along with others utilized later, viz. thioglycerol (Gomori, 1956), $n$-dodecyl mercaptan (Bahr, 1966), benzyl mercaptan (Swift, 1967) and dithiothreitol (Sippel, 1971) as well as 1,4-dithiobutane, its inexpensive but malodourous analogue. 2-Propanol was included in the $\mathrm{pH} 8.5$ buffer to dissolve the water insoluble compounds but probably had the benefit of accelerating reduction of the bonds in keratins (Maclaren, 1962) and similar proteins. The pertinent conclusion from this study is that even at room temperature all of the reagents will eventually reduce disulphides in sections to the same extent.

That these reductions are essentially complete except for the peculiarly resistant bonds in pancreatic zymogen granules is inferred firstly from the otherwise comparable action of TBP. This reagent acts by a different mechanism and is an excellent reductant of disulphide bonds in wool keratins (references in Friedman, 1973). Secondly, dithiothreitol and dithiobutane both should reach favourable equilibria because of their cyclic disulphide products (Cleland, 1964); the former, at least, has been shown abundantly to reduce all accessible disulphide bonds in a great variety of undenatured proteins.

The main drawback to the use of dithiothreitol for precise localization of disulphides is the extraneous staining it induces. Inasmuch as the effect is shown also by dithiobutane and BAL, it would appear to result from thiolation, that is, introduction of exogenous thiol groups into the sections, by way of binding of the bis-mercaptans. A likely mechanism is the condensation of one of the two groups through mercaptal formation since the artifact is most pronounced in arterial elastic tissue which, in young rats, contains free aldehyde groups (MacCallum, 1973); to this extent it resembles the phenomenon encountered during reduction by ammonium sulphide (Barrnett \& Seligman, 1954). More troublesome is the non-specific cytoplasmic staining that in liver cells, for example, is at least equal in magnitude to the level of true disulphide staining. If representing condensation with bound formaldehyde, the error is an unavoidable consequence of formalin-mercurial fixation which presently seems necessary for preserving all of the native thiol groups in paraffin sections.

The potential for much more serious thiolation of sections during the application of freshly alkalinized thioglycolic acid apparently has not been recognized beyond the briefest mention (Sippel, 1969). It can proceed through the acylation of protein amino groups by the thioester bonds of polymeric thioglycolides long known to accumulate in the stored reagent (cf. White, 1960). None are present in dry sodium thioglycolate which, however, has a short shelf life (Lillie, 1965; Sippel, unpublished work) and has not come into general use. Although the offending bonds possibly become hydrolyzed in the hot solutions usually employed in histochemical reductions, they clearly pose a problem at room temperature unless the reagent is purified and stored carefully.

Contrary to predictions based on a less sensitive criterion (Sippel, 1973), all of the mercaptans studied tend to reverse the alkylation of native thiol groups by APM 
(subsequently labelled), as well as by non-chromogenic maleimides and iodoacetate. Although TBP alters the thiol staining to about the same extent as a non-thiolating mercaptan, it has the clear advantage of reversing colourless alkylation less than any mercaptan. Furthermore, apart from its slow action against the disulphide bonds in highly membranous structures into which entry of the relatively large molecule may be impeded, TBP reduces as thoroughly and nearly as rapidly as dithiothreitol without the possiblity of thiolating. It should be useful, therefore, not only for selective staining of disulphides but also for demonstrating them 'by difference'. Among its convenient properties are good stability in storage and in working solutions which are easily prepared. TBP has a garlic-like odour which is far more tolerable than the stench of benzyl mercaptan but, although not known to be hazardous, is most prudently used with good ventilation since its parent compound (phosphine) is toxic.

With either type of reagent, when reduction proceeds at room temperature for little longer than needed to reach completion, the accompanying reversal is so slight that it would have gone undetected but for the sensitivity of positive staining afforded by the re-reduction test. In appropriate form, this procedure could be used to show on a control section the combined effects of all sources of non-specificity in the selective staining of disulphides: reversal, thiolation (if present) and the reaction of APM with tissue constituents other than the thiol groups generated by reduction. The last of these already is minimized in single staining of disulphides by the exhaustive alkylation that precedes reduction, but even in dual staining it is significant in only the structures in which native thiols are incompletely stained. Thiolation would be underestimated only if the first reductant applied were thiolating, thereby decreasing the number of binding sites for the second. And the reversal component would be overestimated to the extent that the alkylated groups include thiols derived from disulphides along with those already present. However, this error is negligible where the effect of reversal is most important, that is, in demonstrating low levels of disulphides in regions rich in thiols.

When native thiols are to be visualized, on the other hand, the specificity of APM is the only critical factor. In this case, estimating the extent of non-specific staining requires prior establishment of an entirely specific and complete blockade of the thiol groups. It is probably only these that are affected by formamidine disulphide which rapidly converts them to mixed disulphides (Friedman, 1973). However, the imperfect stability of the product slightly exaggerates (in structures rich in thiols) the magnitude of non-specific staining and search for a more nearly ideal test of specificity is continuing. Meanwhile it is felt that the staining procedures devised in the light of the present studies and presented in the following communication will demonstrate reliably either thiols or disulphides in sections.

\section{Acknowledgement}

This work was initiated under Research Grant EY00235 and Special Fellowship EY52557 from the National Eye Institute of the U.S. Public Health Service. 


\section{References}

BAHR, G. F. (1966). Introduction to the cytochemistry of sulfhydryl and disulfide groups. In: Introduction to Quantitative Cytochemistry (ed. G. L. Wied), pp. 469-87. New York: Academic Press

BARRNETT, R. J. \& SELIGMAN, A. M. (1954). Histochemical demonstration of sulfhydryl and disulfide groups of proteins. J. natn. Cancer Inst. 14, 769-803.

CLELAND, W. W. (1964). Dithiothreitol, a new protective reagent for SH groups. Biochemistry, N.Y. 3, 480-2.

COWDEN, R. R. \& CURTIS, S. K. (1975). Microspectrophotometric estimates of non-histone proteins in cell nuclei displaying differing degrees of chromatin condensation. J. Morph. 145, 1-12.

DANIELLI, J. F. (1950). Studies on the cytochemistry of proteins. Cold Spring Harb. Symp. quant. Biol. 14, 32-9.

FIERZ-DAVID, H. E. \& BLANGEY, L. (1949). Fundamental Processes of Dye Chemistry. New York: Interscience.

FORAKER, A. G. \& WINGO, W. J. (1956). Protein bound sulfhydryl and disulfide groups in squamous-cell carcinoma of the uterine cervix. Am.J. Obstet. Gynec. 71, $1182-8$.

FRIEDMAN, M. (1973). The Chemistry and Biochemistry of the Sulfhydryl Group in Amino Acids, Peptides and Proteins. Oxford: Pergamon.

GOMORI, G. (1956). Histochemical methods for protein-bound sulfhydryl and disulfide groups. Q. Jl microsc. Sci. 97, 1-9.

KANAOKA, Y., MACHIDA, M., MACHIDA, M. \& SEKINE, T. (1973). Fluorescent thiol reagents. VI. N-(1-anilinonaphthyl-4)maleimide; a fluorescent hydrophobic probe directed to thiol groups in protein. Biochim. biophys. Acta 317, 563-8.

LIL LIE, R. D. (1965). Histopathologic Technic and Practical Histochemistry. 3rd Edn. New York: McGraw-Hill.

MACCALLUM, D. K. (1973). Positive Schiff reactivity of aortic elastin without prior $\mathrm{HIO}_{4}$ oxidation; influence of maturity and a suggested source of the aldehyde. Stain Technol. 48, 117-22.

MACLAREN, J. A. (1962). The extent of reduction of wool proteins by thiols. Aust. J. Chem. 15, 824-31.

PEARSE, A. G. E. (1968). Histochemistry, Theoretical and Applied. 3 rd Edn., Vol. 1. Boston: Little, Brown.

SAVILLE, B. (1958). A scheme for the colorimetric determination of microgram amounts of thiols. Analyst, Lond. 83, 670-2.

SIPPEL, T. O. (1969). Histochemical demonstration of disulfide sites with a new colored alkylating reagent for sulfhydryl. Anat. Rec. 163, 265.

SIPPEL, T. O. (1971). Histochemistry of protein sulfhydryl and disulfide groups in the lens. Expl. Eye Res. 11,383-8.

SIPPEL, T. O. (1973). The histochemistry of thiols and disulphides. I. The use of $\mathrm{N}$-(4-aminophenyl)maleimide for demonstrating thiol groups. Histochem. J. 5, 413-23.

SWIFT, J. A. (1967). The electron histochemistry of cystine-containing proteins in thin transverse sections of human hair. $J l R$. microsc. Soc. 88, 449-60.

TOENNIES, G. (1937). Relations of thiourea, cysteine, and the corresponding disulfides. J. biol. Chem. 120, 297-313.

WHITE, F. H. (1960). Regeneration of enzymatic activity by air-oxidation of reduced ribonuclease with observations on thiolation during reduction with thioglycolate. $J$. biol. Chem. 235, 383-9. 